\title{
Occurrence of second primary malignancies in patients with neuroendocrine tumors of the digestive tract and pancreas
}

\author{
Kimberly Kamp, Ronald A M Damhuis ${ }^{1}$, Richard A Feelders and \\ Wouter $W$ de Herder
}

Department of Internal Medicine, Sector of Endocrinology, Erasmus MC, S-Gravendijkwal 230, 3015 CE Rotterdam,

The Netherlands

${ }^{1}$ Comprehensive Cancer Centre Netherlands, Rotterdam, The Netherlands

(Correspondence should be addressed to W W de Herder; Email: w.w.deherder@erasmusmc.nl)

\begin{abstract}
An increased association between neuroendocrine tumors of the gastrointestinal tract and pancreas (GEP-NET) and other second primary malignancies has been suggested. We determined whether there is indeed an increased risk for second primary malignancies in GEP-NET patients compared with an age- and sex-matched control group of patients with identical malignancies. The series comprised 243 men and 216 women, diagnosed with a GEP-NET between 2000 and 2009 in a tertiary referral center. The timeline, before-at-after diagnosis, and the type of other malignancies were studied using person-year methodology. Poisson distributions were used for testing statistical significance. All data were cross-checked with the Dutch National Cancer Registry. Out of 459 patients with GEP-NET, 67 (13.7\%) had a second primary cancer diagnosis: 25 previous cancers $(5.4 \%), 13$ synchronous cancers $(2.8 \%)$, and 29 metachronous cancers $(6.3 \%)$. The most common types of second primary cancer were breast cancer $(n=10)$, colorectal cancer $(n=8)$, melanoma $(n=6)$, and prostate cancer $(n=5)$. The number of patients with a cancer history was lower than expected, although not significant ( $n=25$ vs $n=34.5)$. The diagnosis of synchronous cancers, mainly colorectal tumors, was higher than expected ( $n=13 \mathrm{vs}$ $n=6.1, P<0.05)$. Metachronous tumors occurred as frequent as expected ( $n=29$ vs $n=25.2$, NS). In conclusion, our results are in contrast to previous studies and demonstrate that only the occurrence of synchronous second primary malignancies, mainly colorectal cancers, is increased in GEP-NET patients compared with the general population.
\end{abstract}

Endocrine-Related Cancer (2012) 19 95-99

\section{Introduction}

Neuroendocrine tumors (NETs) are a heterogeneous group of tumors with various clinical manifestations and biological behavior (Modlin et al. 2008).

The primary localizations of the majority of metastatic NETs are the gastrointestinal (GE) and bronchopulmonary tracts, and pancreas. In addition, these tumors can also be found in other more rare primary localizations such as ovaries, liver, and kidneys.

NETs that originate from cells of the diffuse neuroendocrine system of the gastrointestinal (GI) tract and the pancreas, gastroenteropancreatic NETs (GEP-NETs), are considered to be relatively rare tumors. However, more recent studies on NET epidemiology have demonstrated an increasing GEPNET incidence and prevalence over the past 30 years. According to the United States Surveillance, Epidemiology, and End Results (SEER) database and several other European databases, current estimates of GEPNET incidence vary between 2.5 and 5 cases per 100000 population (Yao et al. 2007, Halfdanarson et al. 2008a,b, Yao et al. 2008, Niederle et al. 2010, Lawrence et al. 2011). It is not yet evident whether this is a true increase in NET incidence, or the result of an increased use of diagnostic procedures, or a combination of both. 
Previously published studies have reported an association between GEP-NETs and second primary 1995, Habal et al. 2000). Unfortunately, these studies were either small case series or autopsy studies (Gerstle et al. 1995, Schneider et al. 1995, Habal et al. 2000). Most studies also did not differentiate between previous, synchronous and metachronous lesions. The absence of age and sex correlations between the investigated populations and National Cancer Registries is also a major drawback in the reported series. Etiologic explanations ranged from incidental discovery to stimulation of cancer growth by neuroendocrine factors.

The aim of this study was to determine whether there was indeed a true increased risk for a second primary malignancy in a GEP-NET patient group compared with an age- and sex-matched control group of patients with identical malignancies.

\section{Patients and methods}

\section{Patients}

Patients with GEP-NETs were identified from the Erasmus MC NET database. Patients diagnosed with the multiple endocrine neoplasia type 1 (MEN1) syndrome were excluded from the study. The medical histories of 459 (non-MEN1) patients with GEP-NET, evaluated between 2000 and 2009 in the Erasmus MC, Rotterdam, The Netherlands, were reviewed. All GEP-NET patients treated in the Erasmus MC, Rotterdam (as described in this study) gave written informed consent before inclusion in the study, which was approved by the Medical Ethics Committee of the Erasmus MC, Rotterdam. Data were collected from medical records and cross-checked with the Dutch National Cancer Registry. The diagnosis of associated second primary malignancies was made by chart review - including pathology reports - the medical history, physical examination of the patient, clinical notes and the correspondence of the referring physician, documenting and cross-checking the previous diagnosis of malignancy.

Associated malignancies were assigned as 'previous' (diagnosed $>6$ months before GEP-NET diagnosis), 'synchronous' (diagnosed within 6 months before or after GEP-NET diagnosis), or 'metachronous' second primary malignancies (diagnosed $>6$ months after GEP-NET diagnosis).

Noninvasive, benign tumors (adenomas), carcinoma in situ of the cervix, and nonmelanoma tumors of the malignancies (Gerstle et al. 1995, Schneider et al.

skin (basaliomas and basal cell cancers) were excluded from this study.

\section{Statistical analysis}

The expected number of second primary malignancies was calculated with age- and sex-specific reference tables, using actuarial calculations (Breslow \& Day 1987). Confidence intervals were constructed using Poisson tables for the observed number of malignancies.

For previous cancers, the age- and sex-specific distribution of the NET cohort was multiplied with a prevalence table, derived from the Dutch National Cancer Registry. The prevalence table describes the proportion of patients living with a previous diagnosis of cancer at a given age and stratified by sex.

For synchronous tumors, person-years at risk were calculated in a similar fashion up to 6 months after diagnosis, and then multiplied by two. The expected number of tumors was obtained by multiplying these person-years at risk with corresponding age- and sexspecific incidence rates for the Dutch population, derived from the Dutch National Cancer Registry.

Table 1 Characteristics of 459 consecutive patients with gastroenteropancreatic neuroendocrine tumors, diagnosed from 2000 to 2009 in the Erasmus MC, Rotterdam, The Netherlands

\begin{tabular}{|c|c|c|}
\hline & $n$ & Percentage (\%) \\
\hline Total & 459 & 100 \\
\hline \multicolumn{3}{|l|}{ Sex } \\
\hline Male & 243 & 52.9 \\
\hline Female & 216 & 47.1 \\
\hline \multicolumn{3}{|l|}{ Age (years) } \\
\hline$<50$ & 63 & 13.7 \\
\hline $50-69$ & 272 & 59.3 \\
\hline$>70$ & 124 & 27.0 \\
\hline \multicolumn{3}{|l|}{ Primary localization } \\
\hline Pancreas & 166 & 36.2 \\
\hline Non-functioning & 130 & 28.3 \\
\hline Insulinoma & 25 & 5.4 \\
\hline Glucagonoma & 1 & 0.2 \\
\hline Gastrinoma & 5 & 1.1 \\
\hline VIPoma & 5 & 1.1 \\
\hline Small intestine & 140 & 30.5 \\
\hline Colorectal & 57 & 12.4 \\
\hline Stomach & 12 & 2.6 \\
\hline Appendix & 6 & 1.3 \\
\hline $\begin{array}{l}\text { Carcinoid } \\
\text { unknown primary } \\
\text { (CUP) }\end{array}$ & 78 & 17.0 \\
\hline \multicolumn{3}{|l|}{ ENETS stage } \\
\hline I-IIIa & 28 & 6.1 \\
\hline IIIb & 26 & 5.7 \\
\hline IV & 405 & 88.2 \\
\hline
\end{tabular}


For metachronous tumors, person-years at risk were calculated from 6 months after the date of diagnosis of the first GEP-NET until the censored date of metachronous cancer, date of death or end of the follow-up (01-01-2010). For the total number of previous, synchronous, and metachronous tumors, differences between the observed and expected numbers were tested for significance using Poisson tables. To avoid post-hoc bias, subgroup analyses were only performed for the most prevalent previous, synchronous, or metachronous second primary malignancies ( $n>3$ per group).

\section{Results}

From 2000 to 2009 , 459 consecutive patients -243 men and 216 women (female-to-male ratio, 1.1:1) - with GEP-NETs were evaluated at the Erasmus MC, Rotterdam, The Netherlands. The median age of the patients at the time of the GEP-NET diagnosis was 62.3 years (range 23.8-89.1 years). The mean follow-up of the study population was 44 months (range 0.4-118.6 months). Table 1 shows the clinical characteristics of the individuals in the analysis. Metastases were demonstrated in 432 patients $(94.1 \%)$. The great majority of patients $(88.2 \%)$ were diagnosed with ENETS stage IV disease (Table 1; Rindi et al. 2006, 2007).

Sixty-three (13.7\%) GEP-NET patients had 67 second primary cancers. Table 2 shows the occurrence of the most prevalent second primary malignancies in 459 patients diagnosed with GEP-NETs divided into previous, synchronous, and metachronous cancers.
The 67 second primary malignancies could be divided over 25 previous cancers $(5.4 \%), 13$ synchronous cancers $(2.8 \%)$, and 29 metachronous cancers $(6.3 \%)$. The most common types of second primary cancer were breast cancer $(n=10)$, colorectal cancer $(n=8)$, melanoma $(n=6)$, and prostate cancer $(n=5$; Table 2$)$. Other second primary malignancies tumors, which are not included in the table, because of their small numbers, were: bronchial carcinoma $(n=2)$, small intestinal carcinoma $(n=2)$, renal cell carcinoma $(n=4)$, lung carcinoma $(n=2)$, gynecological malignancies $(n=3)$, myelodysplastic syndromes $(n=2)$, and leukemia $(n=2)$.

The number of patients with a cancer history was lower than expected but not significantly ( $n=25$ vs $n=34.5)$. Diagnosis of synchronous cancers was higher than expected ( $n=13$ vs $n=6.1, P<0.05$ ). Synchronous cancers were colorectal cancer $(n=4)$, small intestinal cancer $(n=2)$, bronchial carcinoma $(n=2)$, renal cell cancer $(n=2)$, breast cancer $(n=1)$, prostate cancer $(n=1)$, and bladder cancer $(n=1)$. Metachronous tumors occurred as frequent as expected $(n=29$ vs $n=25.2, \mathrm{NS})$.

\section{Discussion}

We have evaluated the occurrence of second primary malignancies in a large cohort of patients with GEP-NETs, who were followed in a single, academic, tertiary referral institution. We have only found a significant increased risk of synchronous second

Table 2 Occurrence of second primary malignancies in 459 patients with gastroenteropancreatic neuroendocrine tumors (GEP-NETs), diagnosed from 2000 to 2009 in the Erasmus MC, Rotterdam, The Netherlands

\begin{tabular}{|c|c|c|c|c|c|}
\hline & Observed & Expected & $95 \% \mathrm{Cl}$ & SIR (O/E) & $95 \% \mathrm{Cl}(\mathrm{SIR})$ \\
\hline \multicolumn{6}{|l|}{ Prev } \\
\hline Total & 25 & 34.5 & $16.2-36.9$ & 0.72 & $0.47-1.07$ \\
\hline Prostate & 5 & 6.7 & $1.6-11.7$ & 0.75 & $0.24-1.75$ \\
\hline Breast & 5 & 7.5 & $1.6-11.7$ & 0.67 & $0.21-1.56$ \\
\hline Melanoma & 4 & 2.3 & $1.1-10.2$ & 1.74 & $0.48-4.43$ \\
\hline \multicolumn{6}{|l|}{ Synchr } \\
\hline Total & 13 & 6.1 & $6.9-22.2^{*}$ & 2.13 & $1.13-3.64$ \\
\hline Colorectal & 4 & 0.9 & $1.1-10.2^{*}$ & 4.44 & $1.22-11.33$ \\
\hline \multicolumn{6}{|l|}{ Metachr } \\
\hline Total & 29 & 25.2 & $19.4-41.7$ & 1.15 & $0.77-1.65$ \\
\hline Breast & 5 & 2.6 & $1.6-11.7$ & 1.92 & $0.62-4.50$ \\
\hline Colorectal & 4 & 4.0 & $1.1-10.2$ & 1.00 & $0.28-2.55$ \\
\hline Total & 67 & 65.8 & $51.9-85.0$ & 1.02 & $0.79-1.29$ \\
\hline
\end{tabular}

Prev (previous second primary malignancies), diagnosed $>6$ months before GEP-NET diagnosis; Synchr (synchronous second primary malignancies), diagnosed within 6 months before or after GEP-NET diagnosis; Metachr (metachronous second primary malignancies), diagnosed $>6$ months after GEP-NET diagnosis; $\mathrm{Cl}$, confidence interval; SIR, standardized incidence ratio. ${ }^{\star} P<0.05$. 
primary malignancies, mainly colorectal cancers, in patients with GEP-NET.

We have chosen not to use GEP-NET data from a National Registry since it occurred to us that the GEP-NET registration in the Dutch National Cancer Registry is incomplete. Reasons for this decision were: some GEP-NETs were not considered to be malignant and, therefore, not reported. Variability in the GEP-NET nomenclature occurred over time. Also variability in classification systems was noted over time. In our study group, the great majority of patients were diagnosed with ENETS stage IV disease. Patients were not randomized.

The Netherlands has an estimated population of 16.6 million people. Our center covers approximately onefifth of this population. Until now, there is no national GEP-NET registry in The Netherlands. Therefore, we cannot give an impression on the proportion of Dutch GEP-NET patients who are treated in our center.

In historical series, the incidence of second primary malignancies in patients with GE-NETs (carcinoids) ranged from 12 to $46 \%$, with an average of $17 \%$ (Habal et al. 2000). In our series, the incidence of second primary malignancies in patients with GEP-NETs is $13.7 \%$, which is in line with the findings in these historical GE-NET series.

A different distribution of GE-NETs (carcinoids) was noted in Taiwanese patients. In comparison with Western patients with GE-NETs, the Taiwanese patients presented with significantly more carcinoid tumors located in the rectum ( $\mathrm{Li}$ et al. 2008). This study showed that Taiwanese GE-NET patients had a high probability of developing associated, noncarcinoid tumors mainly in the GE tract, lungs, and the genitourinary system. However, a statistical quantification of risk using a national reference group was not performed (Li et al. 2008).

It still remains questionable whether there is a true increased incidence of second primary malignancies in GEP-NET patients. The historical series did not correct for age, sex, period of diagnosis, and time from diagnosis and did not provide standardized incidence/ mortality ratios, nor used data obtained from national cancer registries for comparison. Population-based cancer registries can provide high-quality, long-term, national data, with histological confirmation in the majority of cases (Tulinius et al. 1992). The major strength of our study is the use of an age and sex national reference group by using linkage to the Dutch National Cancer Registry.

In a study on NETs (carcinoids) and adenocarcinomas of the small intestine, Zar et al. (2008) corrected their analyses for sex, age, period of diagnosis, and time from diagnosis. These authors concluded that second primary malignancies were generally diagnosed within the first year after the diagnosis of a tumor in the small intestine. This was possibly due to the extensive clinical work-up and follow-up of their patients (Zar et al. 2008).

In a study with a similar design in patients with primary lung carcinoids, Cote et al. (2006) reported an increased risk of breast cancer in females within the first 5 years after the diagnosis of the lung carcinoid. However, after that period, the risk of breast cancer was lower than expected (Cote et al. 2006). These authors also reported on increased risks of breast and prostate cancer in males who had an earlier diagnosis of a lung carcinoid. In these studies, other types of second primary malignancies in lung carcinoid patients were not more prevalent than in the general population (Cote et al. 2006).

Statistical quantification of risk using a populationbased reference group has not yet been used for analyzing second primary cancer risks in GEP-NET patients. Therefore, we have conducted an analysis in this group of patients, using the same methodology as the study in patients with lung carcinoids (Cote et al. 2006). Our methodology was also similar to the methodologies used in two large studies analyzing second primary cancer risks in patients with Merkel cell carcinomas, which are neuroendocrine skin tumors (Kaae et al. 2010, Bzhalava et al. 2011).

In conclusion, our results are refining conclusions obtained in previous studies and demonstrate that mainly the occurrence of synchronous second primary (intestinal) malignancies is increased in GEP-NET patients compared with the general population. This is probably due to incidental findings obtained at radiological or surgical examination, or gastroenterology work-up. Surveillance bias after diagnosis should always be considered as an explanation for excess risk of second primary malignancies, as medical attention is intensified immediately after a cancer diagnosis.

Owing to the rarity of GEP-NET and the diversity of the other cancer types, collaborative international studies will be required to study this issue in further detail. This study does not support extensive screening programs for second primary malignancies in GEP-NET patients.

\section{Declaration of interest}

The authors declare that there is no conflict of interest that could be perceived as prejudicing the impartiality of the research reported. 


\section{Funding}

This research did not receive any specific grant from any funding agency in the public, commercial or not-for-profit sector.

\section{Author contribution statement}

R A Feelders, W W de Herder, and K Kamp collected the patient group data and wrote the manuscript. K Kamp and R A M Damhuis collected the control group data and performed the data/statistical analysis. K Kamp performed the data registry.

\section{References}

Breslow NE \& Day NE 1987 The analysis of cohort studies. Statistical Methods in Cancer Research, vol 2. Lyon, France: IARC Scientific Publications.

Bzhalava D, Bray F, Storm H \& Dillner J 2011 Risk of second cancers after the diagnosis of Merkel cell carcinoma in Scandinavia. British Journal of Cancer 104 178-180. (doi:10.1038/sj.bjc.6605989)

Cote ML, Wenzlaff AS, Philip PA \& Schwartz AG 2006 Secondary cancers after a lung carcinoid primary: a population-based analysis. Lung Cancer 52 273-279. (doi:10.1016/j.lungcan.2006.02.004)

Gerstle JT, Kauffman GL Jr \& Koltun WA 1995 The incidence, management, and outcome of patients with gastrointestinal carcinoids and second primary malignancies. Journal of the American College of Surgeons 180 427-432.

Habal N, Sims C \& Bilchik AJ 2000 Gastrointestinal carcinoid tumors and second primary malignancies. Journal of Surgical Oncology 75 310-316. (doi:10.1002/10969098(200012)75:4<306::AID-JSO14>3.0.CO;2-3)

Halfdanarson TR, Rabe KG, Rubin J \& Petersen GM 2008a Pancreatic neuroendocrine tumors (PNETs): incidence, prognosis and recent trend toward improved survival. Annals of Oncology 19 1727-1733. (doi:10.1093/annonc/ $\operatorname{mdn} 351$ )

Halfdanarson TR, Rubin J, Farnell MB, Grant CS \& Petersen GM 2008b Pancreatic endocrine neoplasms: epidemiology and prognosis of pancreatic endocrine tumors. Endocrine-Related Cancer 15 409-427. (doi:10.1677/ERC-07-0221)

Kaae J, Hansen AV, Biggar RJ, Boyd HA, Moore PS, Wohlfahrt J \& Melbye M 2010 Merkel cell carcinoma: incidence, mortality, and risk of other cancers. Journal of the National Cancer Institute 102 793-801. (doi:10.1093/ jnci/djq120)

Lawrence B, Gustafsson BI, Chan A, Svejda B, Kidd M \& Modlin IM 2011 The epidemiology of gastroenteropancreatic neuroendocrine tumors. Endocrinology and Metabolism Clinics of North America 40 1-18. (doi:10. 1016/j.ecl.2010.12.005)

Li AF, Hsu CY, Li A, Tai LC, Liang WY, Li WY, Tsay SH \& Chen JY 2008 A 35-year retrospective study of carcinoid tumors in Taiwan: differences in distribution with a high probability of associated second primary malignancies. Cancer 112 274-283. (doi:10.1002/cncr. 23159)

Modlin IM, Oberg K, Chung DC, Jensen RT, de Herder WW, Thakker RV, Caplin M, Delle Fave G, Kaltsas GA, Krenning EP et al. 2008 Gastroenteropancreatic neuroendocrine tumours. Lancet Oncology 9 61-72. (doi:10. 1016/S1470-2045(07)70410-2)

Niederle MB, Hackl M, Kaserer K \& Niederle B 2010 Gastro-entero-pancreatic neuroendocrine tumours - the current incidence and staging based on the WHO and ENETS classification. Endocrine-Related Cancer 17 909-918. (doi:10.1677/ERC-10-0152)

Rindi G, Kloppel G, Alhman H, Caplin M, Couvelard A, de Herder WW, Erikssson B, Falchetti A, Falconi M \& Komminoth P 2006 TNM staging of foregut (neuro)endocrine tumors: a consensus proposal including a grading system. Virchows Archiv 449 395-401. (doi:10.1007/s00428-006-0250-1)

Rindi G, Kloppel G, Couvelard A, Komminoth P, Korner M, Lopes JM, McNicol AM, Nilsson O, Perren A, Scarpa A et al. 2007 TNM staging of midgut and hindgut (neuro)endocrine tumors: a consensus proposal including a grading system. Virchows Archiv 451 757-762. (doi:10.1007/s00428-007-0452-1)

Schneider C, Wittekind C \& Kockerling F 1995 Aussergewöhnliche Häufung von Carcinoidtumoren und Zweitmalignomen. Chirurg 66 607-611.

Tulinius H, Storm HH, Pukkala E, Andersen A \& Ericsson J 1992 Cancer in the Nordic countries, 1981-86. A joint publication of the five Nordic Cancer Registries. Acta Pathologica, Microbiologica, et Immunologica Scandinavica Supplement 31 1-194.

Yao JC, Eisner MP, Leary C, Dagohoy C, Phan A, Rashid A, Hassan M \& Evans DB 2007 Population-based study of islet cell carcinoma. Annals of Surgical Oncology 14 3492-3500. (doi:10.1245/s10434-007-9566-6)

Yao JC, Hassan M, Phan A, Dagohoy C, Leary C, Mares JE, Abdalla EK, Fleming JB, Vauthey JN, Rashid A et al. 2008 One hundred years after 'carcinoid': epidemiology of and prognostic factors for neuroendocrine tumors in 35825 cases in the United States. Journal of Clinical Oncology 26 3063-3072. (doi:10.1200/JCO.2007.15. 4377)

Zar N, Garmo H, Holmberg L \& Hellman P 2008 Risk of second primary malignancies and causes of death in patients with adenocarcinoma and carcinoid of the small intestine. European Journal of Cancer 44 718-725. (doi:10.1016/j.ejca.2007.12.003)

Received in final form 7 December 2011 Accepted 22 December 2011 Made available online as an Accepted Preprint 22 December 2011 